\title{
Effect of Spironolactone on Atrial Fibrillation in Patients with Heart Failure with Preserved Ejection Fraction: Post-Hoc Analysis of the Randomized, Placebo-Controlled TOPCAT Trial
}

\author{
Jolien Neefs ${ }^{1}$ (D) Nicoline W. E. van den Berg ${ }^{1}$. Sébastien P. J. Krul ${ }^{1}$. S. Matthijs Boekholdt ${ }^{1}$. Joris R. de Groot ${ }^{1,2}$
}

Published online: 19 June 2019

(c) The Author(s) 2019

\begin{abstract}
Background Mineralocorticoid receptor antagonists (MRAs) reduce the risk of atrial fibrillation (AF) in patients with heart failure (HF) and a reduced ejection fraction. The efficacy of MRAs for AF prevention in patients with HF and a preserved ejection fraction (HFpEF) is unclear.

Objectives We performed a secondary analysis of a randomized placebo-controlled trial to determine the efficacy of spironolactone in reducing new-onset $\mathrm{AF}$ and recurrence of $\mathrm{AF}$ in 2733 patients with symptomatic HFpEF.

Methods Patients with and without prevalent AF at baseline were included, and those with permanent AF were excluded. Patients were randomized 1:1 to spironolactone or placebo. The risk of new-onset AF or the recurrence of AF was quantified using hazard ratios (HRs) with corresponding 95\% confidence intervals (CIs).

Results At baseline, 2228 (64.7\%) patients had no history of AF (spironolactone, $n=1111$; placebo, $n=1117$ ), whereas $505(18.4 \%)$ patients had prevalent AF (spironolactone, $n=260$; placebo, $n=245$ ). During a median follow-up of 3.1 years (interquartile range [IQR] 2.0-4.9), the incidence of new-onset AF was similar in both treatment arms: spironolactone 5.2\% $(n=58)$ versus placebo $4.4 \%(n=49) ; p=0.41$. The risk of new-onset AF was similar in both treatment arms: HR 1.19; 95\% CI 0.81-1.74; $p=0.38$. AF recurrence was also similar in both treatment arms during a median follow-up of 3.3 years (IQR 1.9-4.7): spironolactone 11.5\% $(n=30)$ versus placebo $11.8 \%(n=29) ; p=1.00$. The risk of recurrence of AF did not differ per treatment arm: HR 0.94; 95\% CI 0.57-1.58; $p=0.83$.

Conclusion Spironolactone does not reduce the risk of new-onset AF or AF recurrence in patients with HFpEF. This is in contrast to results in cohorts of patients with HF and a reduced ejection fraction.

Clinical trial registration ClinicalTrials.gov identifier no. NCT00094302 (TOPCAT).
\end{abstract}

\section{Introduction}

Heart failure (HF) is a recognized risk factor for new-onset atrial fibrillation (AF) and recurrence of AF [1]. Moreover, $\mathrm{AF}$ is the most common arrhythmia in HF independent of left ventricle ejection fraction (LVEF) [2]. The increased

Electronic supplementary material The online version of this article (https://doi.org/10.1007/s40256-019-00353-5) contains supplementary material, which is available to authorized users.

Joris R. de Groot

j.r.degroot@amc.uva.nl

1 Department of Cardiology, Heart Center, Academic Medical Center, Amsterdam, The Netherlands

2 Department of Cardiology, Amsterdam UMC, University van Amsterdam, Meibergdreef 9, 1105 AZ Amsterdam, The Netherlands risk of AF in patients with HF can be partly explained by enhanced activation of the renin-angiotensin-aldosterone system (RAAS) and subsequent aldosterone production [1, 3].

Aldosterone competitively binds to the mineralocorticoid receptor, initiating — among other effects-structural cardiac remodeling, a process driven by fibrosis formation $[4,5]$. Like HF, AF is characterized by structural atrial remodeling due to atrial fibrosis [6]. Consequently, aldosterone pathway blockade by mineralocorticoid receptor antagonists (MRAs), such as spironolactone and eplerenone, may reduce HF symptoms and the risk of AF. MRAs were found to be effective in reducing new-onset AF or recurrence of pre-existent $\mathrm{AF}$ in patients with HF, not further specified [7]. Moreover, a secondary analysis of the EMPHASIS-HF (Eplerenone in Mild Patients Hospitalization And SurvIval Study in Heart Failure) trial, which included only patients with HF with a 


\section{Key Points}

Atrial fibrillation (AF) is a common comorbidity in patients with symptomatic heart failure with a preserved ejection fraction.

Spironolactone treatment did not reduce the risk of new-onset $\mathrm{AF}$ or recurrence of $\mathrm{AF}$ in patients with heart failure and a preserved ejection fraction.

Specifically, in patients with comorbidities related to an increased risk of $\mathrm{AF}$, such as hypertension and obesity, spironolactone did not reduce new-onset AF or recurrence of AF.

These findings are in contrast to previous findings in patients with symptomatic heart failure with a reduced ejection fraction.

reduced ejection fraction (HFrEF), showed that eplerenone significantly reduced new-onset AF [8, 9].

A post-hoc analysis of the TOPCAT (Treatment of Cardiac Function with an Aldosterone Antagonist; NCT00094302) trial assessed the influence of AF at baseline on HF outcomes. Patients with AF had a higher cardiovascular risk than patients without $\mathrm{AF}$, independent of spironolactone use [10]. However, whether spironolactone has a beneficial effect on the prevention of new-onset AF or recurrence of $\mathrm{AF}$ in patients with $\mathrm{HF}$ and a preserved ejection fraction (HFpEF) is currently unknown. The primary objective of this analysis was to determine the efficacy of spironolactone in patients with HFpEF included in the TOPCAT study in reducing new-onset AF (i.e., AF in patients without a previous history of $\mathrm{AF}$ ) and recurrence of $\mathrm{AF}$ (i.e., $\mathrm{AF}$ in patients with $\mathrm{AF}$ at baseline or patients in sinus rhythm, but with a medical history of AF), separately. Second, the efficacy of spironolactone was determined in subgroups defined by previously recognized AF risk factors.

\section{Methods}

TOPCAT was a phase III, multicenter, international, randomized, double-blind, placebo-controlled trial. A detailed description of the study design and data collection has been previously published $[11,12]$. The trial was approved by each study site ethics committee, and all patients provided written consent before inclusion. In brief, the trial was designed to determine whether spironolactone treatment in patients with HFpEF improved the composite endpoint of death from cardiovascular causes, aborted cardiac arrest or hospitalization for the management of HF. We included all patients in the TOPCAT study in the current analysis and performed subanalysis on the region of inclusion, as this has been suggested to have affected the results of the main study [13].

\subsection{Study Design}

Patients were eligible when diagnosed with symptomatic HF and LVEF $\geq 45 \%$ combined with either a hospitalization for HF within 12 months prior to inclusion or an elevated natriuretic peptide level (brain natriuretic peptide $[\mathrm{BNP}] \geq$ $100 \mathrm{pg} / \mathrm{mL}$ or N-terminal pro-BNP [NT-proBNP] $\geq 360 \mathrm{pg} /$ $\mathrm{mL}$ ) within 60 days prior to inclusion. Patients had to be aged $\geq 50$ years, have controlled systolic blood pressure $<$ $140 \mathrm{mmHg}$ (or $\leq 160 \mathrm{mmHg}$ if the patient was taking three or more medications to control blood pressure), and a serum potassium level $<5.0 \mathrm{mmol} / \mathrm{L}$. The main exclusion criteria were life expectancy $<3$ years, estimated glomerular filtration rate $(\mathrm{eGFR})<30 \mathrm{ml} / \mathrm{min} / 1.73 \mathrm{~m}^{2}$ body surface area or serum creatinine $\geq 2.5 \mathrm{mg} / \mathrm{dL}$. Inclusion and exclusion criteria were described in detail in the main study publication $[11,12]$.

Initially, the TOPCAT investigators determined a positive history of AF from patients' medical charts and baseline electrocardiogram (ECG). This was reported in study case report forms (CRFs). The presence of AF for the current analysis was obtained from the CRFs, which were made available to the investigators by the National Heart, Lung and Blood Institute's Biologic Specimen and Data Repository Information Coordinating Center. For the current analysis, patients with permanent AF were excluded.

Patients were randomized 1:1 to receive spironolactone or placebo. Initial dosage of study drugs was $15 \mathrm{mg}$ once daily, increased to a maximum of $45 \mathrm{mg}$ daily during the first 4 months after randomization, if tolerated and adjusted, if required.

\subsection{Atrial Fibrillation (AF) Ascertainment}

AF was a predetermined secondary outcome of the TOPCAT trial. Patients were followed for a minimum of 15 months to assess primary and secondary outcomes of the TOPCAT trial. During scheduled outpatient clinic visits, AF occurrence was evaluated or obtained from patient medical charts and reported in the CRFs. New-onset AF or recurrence of AF during follow-up was obtained from the CRF specifically designed for registration of AF occurrence [12]. All ECGs or rhythm strips of cases of new-onset or recurrent AF were adjudicated by a critical event committee. 


\subsection{Statistical Analysis}

Baseline characteristics of patients with and without AF at baseline were compared using unpaired sample $t$ tests for continuous variables and using Pearson's $\chi^{2}$ for categorical variables. The primary outcome of the current analysis was the onset of AF or recurrence of prevalent AF. Kaplan-Meier estimates were used to compute the cumulative incidence of $\mathrm{AF}$, and log-rank was used for between-group comparisons. Cox proportional hazards models were used to quantify the risk of new-onset or recurrent AF, and expressed as hazard ratios (HRs) with corresponding 95\% confidence intervals (CIs). Patients who did not have an episode of AF were censored at the date of last available follow-up information. Incidence rates per 1000 person-years were calculated.

Furthermore, the influence of AF at baseline on HF symptoms was determined by assessing the risk of the primary outcome of the TOPCAT trial (composite of death from cardiovascular causes, aborted cardiac arrest or hospitalization for the management of HF) stratified for a history of AF at baseline using Cox proportional hazards models.

To assess the homogeneity of the drug effect, prespecified subgroups from the initial TOPCAT trial were used in Cox proportional hazards models [11].

To assess potential regional differences, sensitivity analyses were conducted using Cox proportional hazards models $[13,14]$. Further sensitivity analyses were conducted to assess the influence of concomitant angiotensin-convertingenzyme (ACE) inhibitors and angiotensin receptor blockers (ARBs).

Data were analyzed using SPSS version 24 (IBM, Armonk, NY, USA) and R 3.3.2. A two-sided $p$ value of $<$ 0.05 was considered to be significant.

\section{Results}

\subsection{Study Population}

A total of 3445 patients were included in the TOPCAT trial. At baseline, 2228 patients (64.7\%) had no history of AF (spironolactone, $n=1111$; placebo, $n=1117$ ), and prevalent AF was present in 505 (14.7\%) patients (spironolactone, $n$ = 260; placebo, $n=245)$ (Table 1). In total, $672(19.5 \%)$ patients had permanent AF so were excluded from the current analysis. Baseline characteristics of patients without a history of AF at baseline were equally distributed between both treatment arms. This also applied to patients with prevalent AF. Beta-blockers, ACE inhibitors and ARBs were extensively prescribed in the TOPCAT trial but similarly in both treatment arms for patients with and without $\mathrm{AF}$ at baseline. There were significant, but clinically moderately relevant, differences in baseline characteristics between patients with prevalent AF and those without AF. Patients with prevalent $\mathrm{AF}$ were older but had lower rates of diabetes mellitus and coronary artery disease. Patients with prevalent $\mathrm{AF}$ at baseline were more frequently eligible for inclusion in the TOPCAT trial because of elevated natriuretic peptides than because of hospitalization for HF. Patients with prevalent $\mathrm{AF}$ at baseline had a significant larger left atrial volume index than patients without a history of AF at baseline (albeit, on average, lower than the upper boundary of normal). Most patients with prevalent AF showed sinus rhythm on their baseline ECG (spironolactone $84.6 \%$ vs. placebo $81.2 \% ; p=0.06$ ). At baseline, $\mathrm{AF}$ was significantly more reported in patients from Russia and Georgia than in patients from the Americas (18.9 vs. $15.5 \%$, respectively; $p=0.047$ ).

After stratification based on history of AF at baseline, spironolactone did not reduce the risk of the primary composite outcome of death from cardiovascular causes, aborted cardiac arrest or hospitalization for the management of HF in patients either with or without a history of AF at baseline (Figs. 1 and 2 in the Electronic Supplementary Material [ESM]).

\subsection{New-Onset AF During Follow-Up}

During a median follow-up of 3.1 years (interquartile range [IQR] 2.0-4.9), new-onset AF occurred in 5.2\% $(n=58)$ of those treated with spironolactone compared with $4.4 \%$ $(n=49)$ of those treated with placebo $(p=0.41)$ (Fig. 1). This yielded an event rate of 15.8 per 1000 person-years for spironolactone and 13.3 for placebo. The event rate, but not the differential efficacy of spironolactone versus placebo, significantly differed by region of inclusion (Americas 7.4\% vs. Russia and Georgia 3.1\%; $p<0.001$ ). The risk of newonset AF was not significantly different between spironolactone and placebo (HR 1.19; 95\% CI 0.81-1.74; $p=0.38$ with log-rank test) (Fig. 2).

Furthermore, subgroup analyses according to subgroups prespecified in the main TOPCAT publication did not reveal any significant study drug effect on new-onset AF (Fig. 3 in the ESM). Importantly, sensitivity analysis based on region of inclusion demonstrated no differences between the regions (Americas, HR 1.02; 95\% CI 0.64-1.64, $p=0.92$; Russia and Georgia, HR 1.53; 95\% CI 0.78-3.01, $p=0.22$ ) (Fig. 3). Concomitant use of ACE inhibitors, ARBs did not alter the results, nor did concomitant use of any $\beta$-blocker (data not shown).

\subsection{Recurrence of AF During Follow-Up}

During a median follow-up of 3.3 years (IQR 1.9-4.7), AF recurred in $11.5 \%(n=30)$ of patients with a history of prevalent $\mathrm{AF}$ treated with spironolactone compared with $11.8 \%(n=29)$ of those treated with placebo $(p=1.00)$ 
Table 1 Baseline characteristics of 2733 patients with and without prevalent atrial fibrillation

\begin{tabular}{|c|c|c|c|c|c|c|c|}
\hline \multirow[t]{2}{*}{ Characteristics } & \multicolumn{3}{|c|}{ Prevalent AF at baseline } & \multicolumn{3}{|l|}{ No AF at baseline } & \multirow[t]{2}{*}{$p$ Value $^{\mathrm{a}}$} \\
\hline & $\begin{array}{l}\text { Spironolactone }(n \\
=260)\end{array}$ & $\begin{array}{l}\text { Placebo }(n= \\
245)\end{array}$ & Total $(n=505)$ & $\begin{array}{l}\text { Spironolactone }(n \\
=1111)\end{array}$ & $\begin{array}{l}\text { Placebo }(n= \\
1117)\end{array}$ & Total $(n=2228)$ & \\
\hline Age, years & $71(63-79)$ & $71(64-79)$ & $71(64-79)$ & $67(60-74)$ & $67(59-74)$ & $67(60-74)$ & $<0.001$ \\
\hline Females & $131(50.4)$ & $130(53.1)$ & $261(51.7)$ & $613(55.2)$ & $601(53.8)$ & $1214(54.5)$ & 0.28 \\
\hline White race & $242(93.1)$ & 227 (92.7) & $469(92.9)$ & $964(86.8)$ & $981(87.8)$ & $1945(87.3)$ & $<0.001$ \\
\hline LVEF, \% & $61(56-64)$ & $59(52-65)$ & $60(54-64)$ & $60(56-64)$ & $61(56-65)$ & $61(56-65)$ & $<0.001$ \\
\hline LAVI, $\mathrm{mL} / \mathrm{m}^{2}$ & $29(22-39)$ & $30(22-35)$ & $29(22-37)$ & $24(19-31)$ & $25(20-31)$ & $25(19-31)$ & $<0.001$ \\
\hline NYHA class & & & & & & & 0.09 \\
\hline I-II & $172(66.2)$ & $163(66.5)$ & $335(66.3)$ & 777 (69.9) & $791(70.8)$ & $1568(70.4)$ & \\
\hline III-IV & $88(33.8)$ & $81(33.1)$ & $169(33.5)$ & $333(30.0)$ & $324(29.0)$ & $657(29.5)$ & \\
\hline $\begin{array}{l}\text { Heart rate, beats/ } \\
\text { min }\end{array}$ & $66(60-73)$ & $66(60-74)$ & $66(60-73)$ & $68(61-75)$ & $68(61-75)$ & $68(61-75)$ & $<0.001$ \\
\hline \multicolumn{8}{|l|}{$\begin{array}{l}\text { Blood pressure, } \\
\mathrm{mmHg}\end{array}$} \\
\hline Systolic & $129(120-135)$ & $130(120-137)$ & $130(120-136)$ & $130(120-140)$ & $130(120-140)$ & $130(120-140)$ & $<0.001$ \\
\hline Diastolic & $76(66-80)$ & $76(70-80)$ & $76(68-80)$ & $80(70-83)$ & $80(70-84)$ & $80(70-84)$ & $<0.001$ \\
\hline BMI, $\mathrm{kg} / \mathrm{m}^{2}$ & $31(27-36)$ & $30(27-35)$ & $31(27-36)$ & $31(27-35)$ & $31(27-36)$ & $31(27-36)$ & $<0.001$ \\
\hline $\mathrm{CAD}^{\mathrm{b}}$ & $139(53.5)$ & $154(62.9)$ & $293(58.0)$ & $685(61.7)$ & $713(63.8)$ & $1398(62.7)$ & 0.05 \\
\hline Hypertension & $240(92.3)$ & $222(90.6)$ & $462(91.5)$ & $1010(90.9)$ & $1038(92.9)$ & 2048 (91.9) & 0.82 \\
\hline Diabetes mellitus & $73(28.1)$ & $57(23.3)$ & $130(25.7)$ & $375(33.8)$ & $384(34.4)$ & $759(34.1)$ & $<0.001$ \\
\hline Eligibility stratum & & & & & & & $<0.001$ \\
\hline $\begin{array}{l}\text { Hospitalization } \\
\text { in previous } \\
\text { year; HF } \\
\text { management a } \\
\text { major compo- } \\
\text { nent }\end{array}$ & $172(66.2)$ & $170(69.4)$ & $342(67.7)$ & $846(76.1)$ & $831(74.4)$ & $1677(75.3)$ & \\
\hline $\begin{array}{l}\text { Elevated NPs } \\
\text { in previous } 60 \\
\text { days }\end{array}$ & $88(33.8)$ & 75 (30.6) & $163(32.3)$ & 265 (23.9) & $286(25.6)$ & $551(24.7)$ & \\
\hline $\begin{array}{l}\text { Region of enroll- } \\
\text { ment }\end{array}$ & & & & & & & $<0.001$ \\
\hline Americas $^{\mathrm{c}}$ & $152(58.5)$ & $128(52.2)$ & $280(55.4)$ & $511(46.0)$ & $511(45.7)$ & $1022(45.9)$ & \\
\hline $\begin{array}{l}\text { Russia and } \\
\text { Georgia }\end{array}$ & $108(41.5)$ & $117(47.8)$ & $225(44.5)$ & $600(54.0)$ & $606(54.3)$ & $1206(54.1)$ & \\
\hline $\begin{array}{l}\text { Serum BNP, pg/ } \\
\text { mL }\end{array}$ & $276(157-542)$ & $267(148-563)$ & $272(149-546)$ & $206(135-487)$ & $224(128-398)$ & $220(131-426)$ & $<0.001$ \\
\hline $\begin{array}{l}\text { Serum NT- } \\
\text { proBNP, pg/mL }\end{array}$ & 784 (503-1634) & $710(441-2034)$ & 784 (480-1877) & $604(382-1165)$ & $698(408-1625)$ & $647(387-1362)$ & $<0.001$ \\
\hline $\begin{array}{l}\text { Serum potassium, } \\
\mathrm{mmol} / \mathrm{L}\end{array}$ & $4.3(4.0-4.6)$ & $4.3(4.0-4.6)$ & $4.3(4.0-4.6)$ & $4.3(4.0-4.6)$ & $4.3(4.0-4.6)$ & $4.3(4.0-4.6)$ & $<0.001$ \\
\hline $\begin{array}{l}\text { Serum creatinine, } \\
\mathrm{mg} / \mathrm{dL}\end{array}$ & $1.1(0.9-1.3)$ & $1.1(0.9-1.2)$ & $1.1(0.9-1.3)$ & $1.0(0.9-1.2)$ & $1.0(0.9-1.2)$ & $1.0(0.9-1.2)$ & $<0.001$ \\
\hline $\begin{array}{l}\text { eGFR, mL/ } \\
\mathrm{min} / 1.73 \mathrm{~m}^{2}\end{array}$ & $61.9(50.9-76.3)$ & $64.0(53.0-77.9)$ & $63.1(52.0-76.9)$ & $67.4(56.1-81.1)$ & $66.2(54.6-80.3)$ & $66.9(55.3-80.6)$ & $<0.001$ \\
\hline $\begin{array}{l}\text { Serum hemo- } \\
\text { globin, g/dl }\end{array}$ & $13.2(11.9-14.2)$ & $13.2(12.4-14.5)$ & $13.2(12.0-14.3)$ & $13.1(12.1-14.2)$ & $13.2(12.1-14.3)$ & $13.1(12.1-14.3)$ & $<0.001$ \\
\hline \multicolumn{8}{|l|}{ Medications } \\
\hline ACE inhibitor & $152(58.5)$ & $149(60.8)$ & 301 (59.6) & $755(68.0)$ & $760(68.0)$ & $1515(68.0)$ & $<0.001$ \\
\hline ARB & $60(23.1)$ & $49(20.0)$ & 109 (21.6) & $212(19.1)$ & 207 (18.5) & 419 (18.8) & 0.17 \\
\hline Aspirin & $155(59.6)$ & $146(59.6)$ & $301(59.6)$ & $805(72.5)$ & 829 (74.2) & $1634(73.4)$ & $<0.001$ \\
\hline$\beta$-blocker & $183(70.4)$ & $169(69.0)$ & $352(69.7)$ & 881 (79.3) & 868 (77.7) & 1749 (78.5) & $<0.001$ \\
\hline $\mathrm{CCB}$ & $94(36.2)$ & $86(35.1)$ & $180(35.6)$ & $418(37.6)$ & 465 (41.6) & 883 (39.6) & 0.11 \\
\hline
\end{tabular}


Table 1 (continued)

\begin{tabular}{|c|c|c|c|c|c|c|c|}
\hline \multirow[t]{2}{*}{ Characteristics } & \multicolumn{3}{|c|}{ Prevalent AF at baseline } & \multicolumn{3}{|l|}{ No AF at baseline } & \multirow[t]{2}{*}{$p$ Value $^{\mathrm{a}}$} \\
\hline & $\begin{array}{l}\text { Spironolactone }(n \\
=260)\end{array}$ & $\begin{array}{l}\text { Placebo }(n= \\
245)\end{array}$ & Total $(n=505)$ & $\begin{array}{l}\text { Spironolactone }(n \\
=1111)\end{array}$ & $\begin{array}{l}\text { Placebo }(n= \\
1117)\end{array}$ & Total $(n=2228)$ & \\
\hline Diuretic & $218(83.8)$ & $205(83.7)$ & $423(83.8)$ & 866 (77.9) & $885(79.2)$ & $1751(78.6)$ & 0.01 \\
\hline $\begin{array}{l}\text { Long-acting } \\
\text { nitrate }\end{array}$ & $37(14.2)$ & $36(14.7)$ & $73(14.5)$ & $180(16.2)$ & $175(15.7)$ & $355(15.0)$ & 0.45 \\
\hline Statin & $146(56.2)$ & $140(57.1)$ & $286(56.6)$ & $584(52.6)$ & $572(51.2)$ & $1156(51.9)$ & 0.06 \\
\hline Warfarin & $119(45.8)$ & $108(44.1)$ & $227(45.0)$ & $59(5.3)$ & $40(3.6)$ & $99(4.4)$ & $<0.001$ \\
\hline
\end{tabular}

Data are presented as $n(\%)$ or (IQR) unless otherwise indicated. Ultrasound data were available in 935 patients

$A C E$ angiotensin-converting-enzyme, $A F$ atrial fibrillation, $A R B$ angiotensin II receptor blocker, $B M I$ body mass index, $B N P$ brain natriuretic peptide, $C A D$ coronary artery disease, $C C B$ calcium channel blocker, $e G F R$ estimated glomerular filtration rate, $H F$ heart failure, $L A V I$ left atrial volume index, $L V E F$ left ventricular ejection fraction, NT-proBNP N-terminal pro-BNP, NYHA New York Heart Association

${ }^{\mathrm{a}}$ No $\mathrm{AF}$ versus prevalent $\mathrm{AF}$

${ }^{\mathrm{b}} \mathrm{CAD}$ includes myocardial infarction, coronary artery bypass graft, percutaneous intervention or angina pectoris

${ }^{\mathrm{c}}$ The Americas included the USA, Canada, Argentina and Brazil
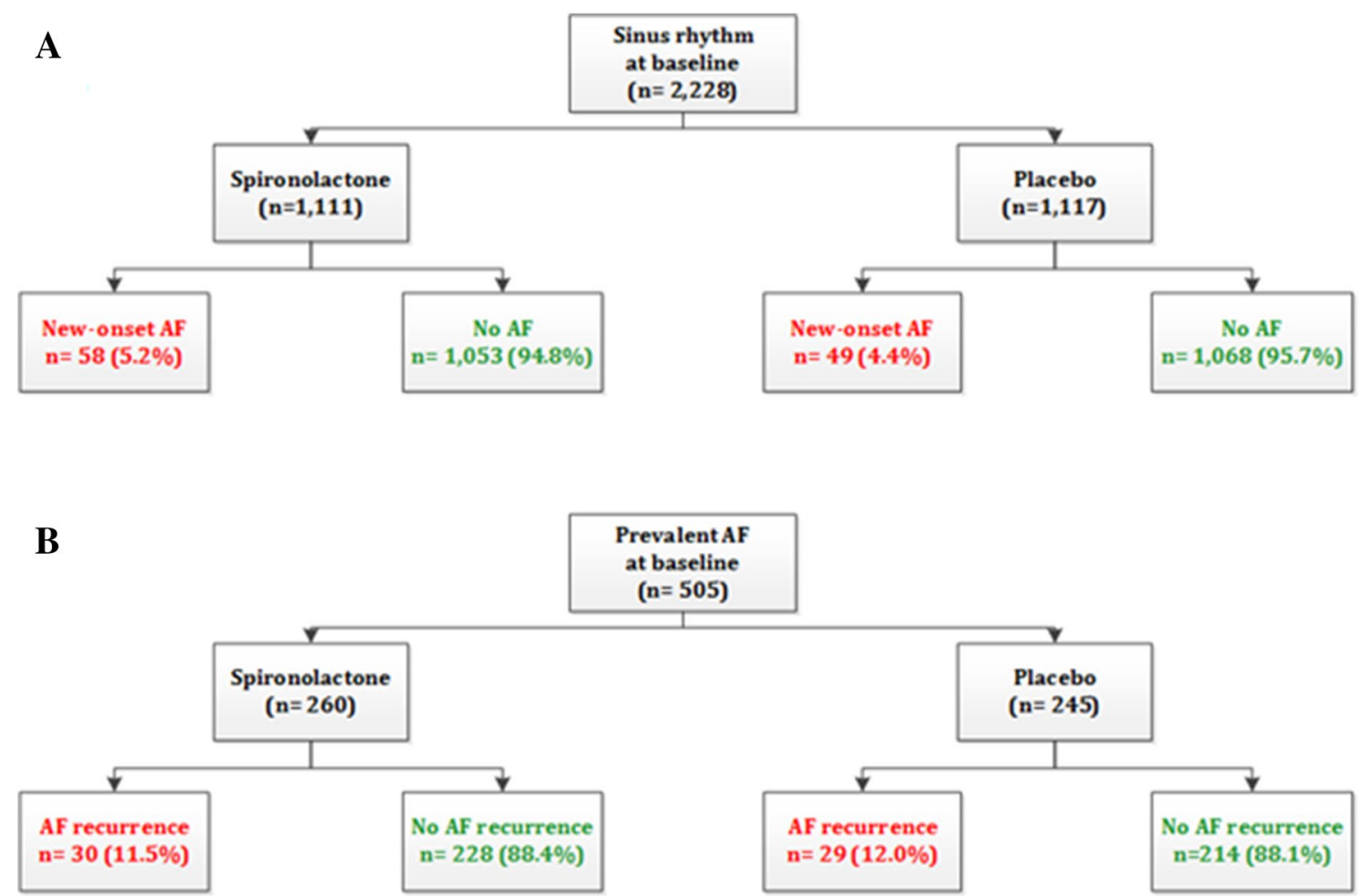

Fig. 1 Cumulative hazard ratio (\%) of a new-onset atrial fibrillation (AF) and $\mathbf{b}$ recurrence of AF for spironolactone versus placebo

(Fig. 1). This yielded an event rate of 35.4 per 1000 personyears for spironolactone and 37.1 for placebo. The rate of AF recurrence was similar per region of inclusion: Americas, $11.6 \%$; Russia and Georgia, $15.4 \% ; p=0.37$. The risk of AF recurrence did not differ between spironolactone- and placebo-treated patients: HR 0.94; 95\% CI 0.57-1.58; $p=$ 0.83 with log-rank test (Fig. 2).

Furthermore, subgroup analyses according to subgroups prespecified in the main TOPCAT publication did not reveal any significant study drug effect on recurrence of AF (Fig. 4 in the ESM). Importantly, sensitivity analysis based on region of inclusion demonstrated no differences between the regions: Americas, HR 0.89; 95\% CI 0.43-1.85; $p=0.76$; Russia and Georgia, HR 0.97; 95\% CI 0.47-2.01; $p=0.93$ (Fig. 3). Concomitant use of ACE inhibitors or ARBs did not alter the results, nor did concomitant use of any $\beta$-blocker (data not shown). 


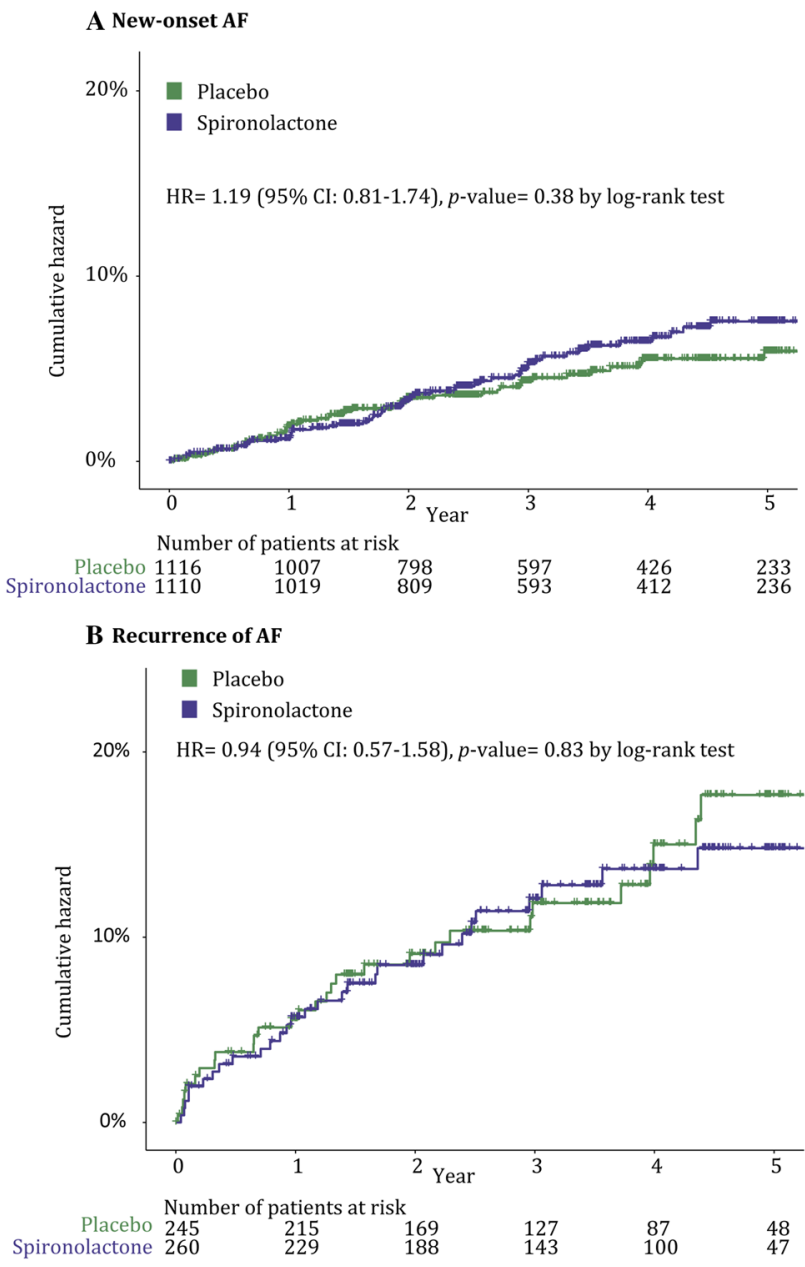

Fig. 2 Number of patients with new-onset AF or recurrence of AF during follow-up for spironolactone versus placebo. $A F$ atrial fibrillation, $C I$ confidence interval, $H R$ hazard ratio

\section{Discussion}

The current secondary analysis of the TOPCAT trial assessed the efficacy of MRAs on the risk of new-onset AF or recurrence of AF separately in patients with HFpEF in a large, randomized, double-blind, placebo-controlled trial. Spironolactone did not reduce new-onset AF or recurrence of AF compared with placebo in patients with symptomatic HFpEF. Moreover, subgroup analyses did not reveal any significant differences. Specifically, in patients with comorbidities related to an increased risk of AF, such as an enlarged left atrium, hypertension and obesity, spironolactone did not reduce new-onset $\mathrm{AF}$ or recurrence of $\mathrm{AF}$. These findings are in contrast to prior findings in patients with HRrEF. In a secondary analysis of the EMPHASIS-HF trial, eplerenone reduced new-onset AF by $42 \%$ compared with placebo [9]. However, we show that spironolactone does not prevent newonset or recurrent $\mathrm{AF}$ in patients with $\mathrm{HFpEF}$, irrespective of region of inclusion or sex. These contradicting results may be explained by either patient- or substrate-related differences.

First, patients with $\mathrm{HFrEF}$ tend to have prevalent coronary artery disease, whereas patients with HFpEF have an underlying risk profile comprising a combination of known cardiac risk factors [2]. Patients from the TOPCAT trial reflect the characteristics of patients with HFpEF as described in the literature, with a very high prevalence of hypertension [15]. Moreover, the incidence of AF differs between HFrEF and $\mathrm{HFpEF}$, in that AF is more common in patients with HFpEF than in those with HFrEF [16]. The current analysis found a relatively low incidence of new-onset AF. However, after comparison of the region of inclusion, event rates in the Americas conformed to those described in the literature, which we discuss later in this article.

Second, the absence of reduction of AF incidence by MRAs in HFpEF may be explained by the distinctive underlying process of cardiovascular remodeling. For example, HFrEF has been associated with degradation and focal fibrosis formation, whereas fibrosis formation in HFpEF is less obvious [17]. Therefore, other processes may prevail in the AF substrate in patients with HFpEF. Importantly, HFpEF is characterized by increased left atrial stiffness and pressure overload, which is thought to contribute to the high burden of AF in patients with HFpEF [18], which MRAs may not particularly affect. Furthermore, it has been suggested that cardiac remodeling in HFpEF can be attributed to systemic inflammation. This, in turn, may lead to both cardiac and noncardiac comorbidities, involving myocardial microvascular dysfunction, leading to myocardial remodeling

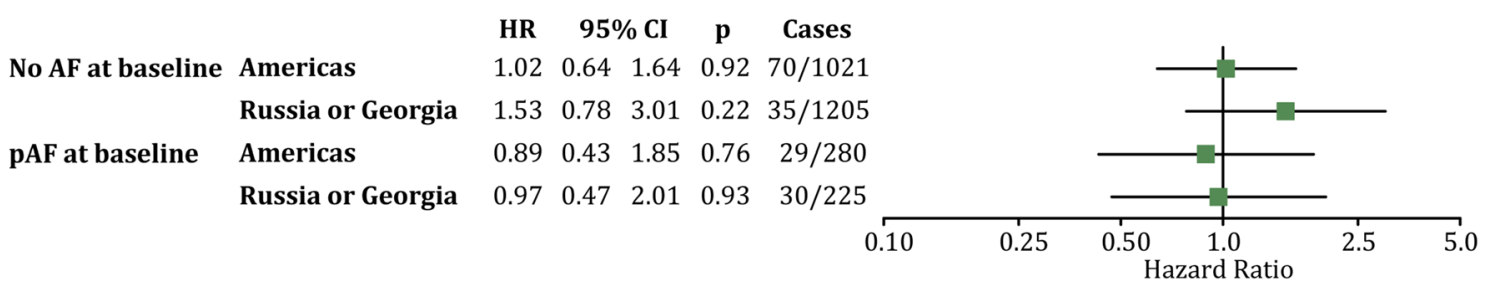

Fig. 3 Forest plot of the hazard ratios of new-onset AF or recurrence of AF stratified for region of inclusion. The Americas included the USA, Canada, Argentina and Brazil. $A F$ atrial fibrillation, $C I$ confidence interval, $H R$ hazard ratio, $p p$ value 
and dysfunction $[17,19]$. Although not tested in the current analysis, these processes may have contributed to the arrhythmogenic substrate in subjects participating in the TOPCAT trial.

Third, it can be rationalized that a decrease of AF onset or recurrence in patients with HFrEF may be the result of a decrease in HF outcomes. The lack of an effect of spironolactone on the prevention of new-onset $\mathrm{AF}$ or $\mathrm{AF}$ recurrence in this study may be due to the neutral primary results concerning HF outcomes in the TOPCAT trial. Indeed, TOPCAT investigators stratified patients to AF or no AF at baseline and found no differential effect of spironolactone or placebo on the main study outcomes [10]. This may imply that the effect of MRAs on AF occurrence is dependent on HF symptoms. However, Dabrowski et al. [20] randomized patients with paroxysmal AF without $\mathrm{HF}$ and with a mean ejection fraction of $69 \%$ to spironolactone or no spironolactone. Recurrence of AF was significantly less frequent in patients treated with spironolactone. Importantly, systolic and diastolic blood pressure did not differ between treatment arms [20]. Thus, although an indirect effect of spironolactone on AF via the treatment of HF may well be possible, an additional effect on the AF substrate (i.e., fibrosis formation) seems likely. Interestingly, the RAAS activity has been suggested to play a larger role in HFrEF than in HFpEF [21, 22]. Combining these factors, it is likely that MRAs alter the risk of AF by reducing profibrotic pathways in the atrial wall, which are triggered by RAAS activation and aldosterone production in the setting of HFrEF. Indeed, the concentration of plasma markers of cardiac fibrosis (carboxy- and amino-terminal propeptide of procollagen type-I [PICP and PINP] and type-III [PIIINP]) decreased after administration of MRA in patients with HFrEF [23]. However, a meta-analysis also found a reduction of cardiac fibrosis markers in patients with HFpEF who were administered MRAs [24].

\subsection{Limitations}

Some aspects of our study should be considered when interpreting the results of this secondary analysis. The presence of AF was derived from the specified case study forms, which may have led to incorrect categorization of AF type. It is therefore also possible that the true incidence of AF was underestimated. In particular, events tended to be underreported in patients from Russia and Georgia, as also described for the primary outcome of the TOPCAT trial [11]. However, we cannot exclude the possibility that the $\mathrm{AF}$ cases identified and reported in the CRFs were those demanding physician contact. Therefore, it can be argued that the clinically most relevant $\mathrm{AF}$ episodes are those that are symptomatic and demand physician contact; this limitation pertains to both randomized treatment arms. Further, the TOPCAT investigators reported a significantly lower systolic blood pressure in the spironolactone group during follow-up (mean decrease of $2.2 \mathrm{vs.} 0.2 \mathrm{mmHg}$ for spironolactone and placebo, respectively; $p<0.001$ ) [11]. AF episodes may have been more symptomatic in patients with low blood pressure, but the mean decrease in systolic blood pressure was relatively mild.

Lastly, the trial included patients drawn from two different regions, the Americas and Russia plus Georgia. These regions included patients with different baseline characteristics. In a secondary analysis, the TOPCAT investigators showed a disparate effect of spironolactone. In patients from the Americas, spironolactone significantly reduced the risk of the primary outcome, but this effect was not significant in patients from Russia and Georgia [13]. In patients randomized to spironolactone, the serum level of canrenone (the active metabolite of spironolactone) was significantly more frequently undetectable in patients from Russia and Georgia than in those from the Americas. The investigators concluded that the study results from Russia and Georgia did not reflect the true therapeutic effect of spironolactone [14]. The current analyses were all based on an intent-totreat population. However, our sensitivity analysis focusing on region of inclusion did not demonstrate different results with respect to new-onset $\mathrm{AF}$ or $\mathrm{AF}$ recurrence between both regions. This argues for the inclusion of the patients from Russia and Georgia in the current analysis.

\section{Conclusion}

Spironolactone does not decrease the risk of new-onset AF or recurrence of AF in patients in the TOPCAT study diagnosed with symptomatic HFpEF. Our findings contrast with previous findings in patients with HFrEF. Further effort is needed to find an effective treatment to reduce the risk of $\mathrm{AF}$, since AF episodes are related to increased hospitalization, stroke and mortality rates.

Acknowledgements The authors thank the TOPCAT investigators. This manuscript was prepared using TOPCAT Research Materials obtained from the National Heart, Lung, and Blood Institute (NHLBI) Biologic Specimen and Data Repository Information Coordinating Center and does not necessarily reflect the opinions or views of the TOPCAT study organization or the NHLBI.

\section{Compliance with Ethical Standards}

Funding No sources of funding were used to conduct this study or prepare this manuscript.

Conflicts of Interest Dr. de Groot is a consultant for AtriCure and Daichii Sankyo; has received research grants from AtriCure, Medtronic, Boston Scientific and Abbott and a grant from ZonMW/now (106.146.310) outside the submitted work. Jolien Neefs, Nicoline van 
den Berg, Sébastien Krul and S. Matthijs Boekholdt have no potential conflicts of interest that might be relevant to this work.

Open Access This article is distributed under the terms of the Creative Commons Attribution-NonCommercial 4.0 International License (http://creativecommons.org/licenses/by-nc/4.0/), which permits any noncommercial use, distribution, and reproduction in any medium, provided you give appropriate credit to the original author(s) and the source, provide a link to the Creative Commons license, and indicate if changes were made.

\section{References}

1. Kirchhof P, Benussi S, Kotecha D, Ahlsson A, Atar D, Casadei B, et al. 2016 ESC Guidelines for the management of atrial fibrillation developed in collaboration with EACTS. Eur Heart J. 2016;37(38):2893-962.

2. Ponikowski P, Voors AA, Anker SD, Bueno H, Cleland JG, Coats AJ, et al. 2016 ESC Guidelines for the diagnosis and treatment of acute and chronic heart failure: the Task Force for the diagnosis and treatment of acute and chronic heart failure of the European Society of Cardiology (ESC)Developed with the special contribution of the Heart Failure Association (HFA) of the ESC. Eur Heart J. 2016;37(27):2129-200.

3. Kotecha D, Chudasama R, Lane DA, Kirchhof P, Lip GY. Atrial fibrillation and heart failure due to reduced versus preserved ejection fraction: a systematic review and meta-analysis of death and adverse outcomes. Int J Cardiol. 2016;203:660-6.

4. Lavall D, Selzer C, Schuster P, Lenski M, Adam O, Schafers HJ, et al. The mineralocorticoid receptor promotes fibrotic remodeling in atrial fibrillation. J Biol Chem. 2014;289(10):6656-68.

5. Parviz Y, Iqbal J, Pitt B, Adlam D, Al-Mohammad A, Zannad F. Emerging cardiovascular indications of mineralocorticoid receptor antagonists. Trends Endocrinol Metab. 2015;26(4):201-11.

6. Nattel S, Burstein B, Dobrev D. Atrial remodeling and atrial fibrillation: mechanisms and implications. Circul Arrhythm Electrophysiol. 2008;1(1):62-73.

7. Neefs J, van den Berg NW, Limpens J, Berger WR, Boekholdt SM, Sanders P, et al. Aldosterone pathway blockade to prevent atrial fibrillation: a systematic review and meta-analysis. Int J Cardiol. 2017;231:155-61.

8. Zannad F, McMurray JJ, Krum H, van Veldhuisen DJ, Swedberg $\mathrm{K}$, Shi $\mathrm{H}$, et al. Eplerenone in patients with systolic heart failure and mild symptoms. N Engl J Med. 2011;364(1):11-21.

9. Swedberg K, Zannad F, McMurray JJ, Krum H, van Veldhuisen DJ, Shi $\mathrm{H}$, et al. Eplerenone and atrial fibrillation in mild systolic heart failure: results from the EMPHASIS-HF (Eplerenone in Mild Patients Hospitalization And SurvIval Study in Heart Failure) study. J Am Coll Cardiol. 2012;59(18):1598-603.

10. Cikes M, Claggett B, Shah AM, Desai AS, Lewis EF, Shah SJ, et al. Atrial fibrillation in heart failure with preserved ejection fraction: the TOPCAT trial. JACC Heart Fail. 2018;6(8):689-97.

11. Pitt B, Pfeffer MA, Assmann SF, Boineau R, Anand IS, Claggett $\mathrm{B}$, et al. Spironolactone for heart failure with preserved ejection fraction. N Engl J Med. 2014;370(15):1383-92.

12. Desai AS, Lewis EF, Li R, Solomon SD, Assmann SF, Boineau R, et al. Rationale and design of the treatment of preserved cardiac function heart failure with an aldosterone antagonist trial: a randomized, controlled study of spironolactone in patients with symptomatic heart failure and preserved ejection fraction. Am Heart J. 2011;162(6):966-72.e10

13. Pfeffer MA, Claggett B, Assmann SF, Boineau R, Anand IS, Clausell N, et al. Regional variation in patients and outcomes in the treatment of preserved cardiac function heart failure with an aldosterone antagonist (TOPCAT) trial. Circulation. 2015;131(1):34-42.

14. de Denus S, O'Meara E, Desai AS, Claggett B, Lewis EF, Leclair $\mathrm{G}$, et al. Spironolactone metabolites in TOPCAT - new insights into regional variation. N Engl J Med. 2017;376(17):1690-2.

15. Yancy CW, Lopatin M, Stevenson LW, De Marco T, Fonarow GC. Clinical presentation, management, and in-hospital outcomes of patients admitted with acute decompensated heart failure with preserved systolic function: a report from the Acute Decompensated Heart Failure National Registry (ADHERE) Database. J Am Coll Cardiol. 2006;47(1):76-84.

16. Sartipy U, Dahlstrom U, Fu M, Lund LH. Atrial fibrillation in heart failure with preserved, mid-range, and reduced ejection fraction. JACC Heart Fail. 2017;5(8):565-74.

17. Paulus WJ, Tschope C. A novel paradigm for heart failure with preserved ejection fraction: comorbidities drive myocardial dysfunction and remodeling through coronary microvascular endothelial inflammation. J Am Coll Cardiol. 2013;62(4):263-71.

18. Melenovsky V, Hwang SJ, Redfield MM, Zakeri R, Lin G, Borlaug BA. Left atrial remodeling and function in advanced heart failure with preserved or reduced ejection fraction. Circ Heart Fail. 2015;8(2):295-303.

19. Shah SJ, Kitzman DW, Borlaug BA, van Heerebeek L, Zile MR, Kass DA, et al. Phenotype-specific treatment of heart failure with preserved ejection fraction: a multiorgan roadmap. Circulation. 2016;134(1):73-90.

20. Dabrowski R, Borowiec A, Smolis-Bak E, Kowalik I, Sosnowski C, Kraska A, et al. Effect of combined spironolactone-betablocker $+/-$ enalapril treatment on occurrence of symptomatic atrial fibrillation episodes in patients with a history of paroxysmal atrial fibrillation (SPIR-AF study). Am J Cardiol. 2010;106(11):1609-14.

21. Benedict CR, Johnstone DE, Weiner DH, Bourassa MG, Bittner V, Kay R, et al. Relation of neurohumoral activation to clinical variables and degree of ventricular dysfunction: a report from the Registry of Studies of Left Ventricular Dysfunction. SOLVD Investigators. J Am Coll Cardiol. 1994;23(6):1410-20.

22. Hogg K, McMurray J. Neurohumoral pathways in heart failure with preserved systolic function. Progress Cardiovasc Dis. 2005;47(6):357-66.

23. Zannad F, Alla F, Dousset B, Perez A, Pitt B. Limitation of excessive extracellular matrix turnover may contribute to survival benefit of spironolactone therapy in patients with congestive heart failure: insights from the randomized aldactone evaluation study (RALES). Rales Investigators. Circulation. 2000;102(22):2700-6.

24. Pandey A, Garg S, Matulevicius SA, Shah AM, Garg J, Drazner $\mathrm{MH}$, et al. Effect of mineralocorticoid receptor antagonists on cardiac structure and function in patients with diastolic dysfunction and heart failure with preserved ejection fraction: a meta-analysis and systematic review. J Am Heart Assoc. 2015;4(10):e002137. 